\title{
A Secure Electronic Transaction Payment Protocol Design and Implementation
}

\author{
Houssam El Ismaili ${ }^{1}$, Hanane Houmani ${ }^{2}$, Hicham Madroumi ${ }^{3}$ \\ Architecture of Systems Team - ENSEM, Hassan II University, 8118, Casablanca - Morocco
}

\begin{abstract}
Electronic payment is the very important step of the electronic business system, and its security must be ensured. SSL/TLS and SET are two widely discussed means of securing online credit card payments. Because of implementation issues, SET has not really been adopted by e-commerce participants, whereas, despite the fact that it does not address all security issues, SSL/TLS is commonly used for Internet e-commerce security. The three-domain (3D) security schemes, including 3-D Secure and 3D SET have recently been proposed as ways of improving ecommerce transaction security. Based on our research about SSL, SET, 3D security schemes and the requirements of electronic payment, we designed a secure and efficient E-Payment protocol. The new protocol offers an extra layer of protection for cardholders and merchants. Customers are asked to enter an additional password after checkout completion to verify they are truly the cardholder; the authentication is done directly between the cardholder and card issuer using the issuer security certificate and without involving the third party (Visa, MasterCard).
\end{abstract}

Keywords-E-commerce; Secure Socket Layer (SSL); Secure Electronic Transaction (SET); 3D-Secure

\section{INTRODUCTION}

Electronic commerce or e-commerce provides participants, including consumers and merchants, with a number of benefits, such as convenience and time savings. E-commerce transactions can be categorized into business to business (B2B), business to consumer (B2C), consumer to consumer (C2C), and public/private sectors to government [1]; we focus on $\mathrm{B} 2 \mathrm{C}$ transactions in this paper.

In $\mathrm{B} 2 \mathrm{C}$ transactions, the credit card is the most widely used method of payment for Internet ecommerce transactions. According to an Internet shopping habits survey conducted by Survey.Net (http://www.survey.net), 36\% of Internet users purchase goods by transmitting their credit card number via a secure form; the percentages for other payment methods are significantly lower. Given that the debit/credit card is the primary means for consumers to purchase products or services online, the possible compromise of credit card numbers is a serious threat to the consumer. The E-payment system brings users with higher efficiency, credibility and speeding-up transactions settlement, which reduce the pay risks caused by time lags in handling the bills. However, it also comes with new risks, i.e. security problem of transactions.

The research reported here builds on the electronic payment security; we study the security of e-commerce protocols and we propose a new efficient protocol to ensure a high security for electronic payment transactions.

The objective of our protocol is to provide issuers with the ability to authenticate cardholders during an online purchase without involving the third party VISA or MasterCard. We define a new transaction flow involving cardholder, merchant, payment gateway and card issuer, and allowed parties to identify themselves to each other and exchange information securely using digital certificate. For some implementation reasons, the cardholder is not requested to have his digital certificate, he use the password code to be authenticated by the card issuer.

\section{SeCurity ReQuirements Of E-PAyment}

It goes as follows [2]:

\section{A. Information confidentiality}

All information during the transactions has the request of being kept confidential. For instance, account number and user name may be embezzled by others who have access to them; business opportunity may be lost if order and payment information of your customer's are obtained by competitors. Thus, encryption is required in the E-C information transmission.

\section{B. Data integrity}

E-C should provide medium to identify data integration, ensuring the Web data do not be altered in transmission.

\section{Authentication of participants}

The parts involved may have never met each other. So to make the transaction successful, the first step is to identify the two parts, which is the essential prerequisite of transactions.

\section{Non-repudiation}

The transaction must have such services that enable one party to prevent another party denying having taken a particular action, e.g. sending order/payment information, confirmation of order/payment. Both consumer and merchant also require this service.

\section{E. End-user implementation Requirements}


We focus here on the major barriers causing implementation failures in SET and other protocols including usability, flexibility, affordability, speed of transaction, and interoperability.

- Usability - The system must be easy to implement, including installation. The consumer requires the card issuer and merchant to provide a secure system that is not complex, while the merchant requires the acquirer and security software developers to provide a simple application that meets the security requirements.

- Flexibility - The system must allow e-commerce consumers to order products or services from any location, and not just from one PC. Here, the consumer is the entity requiring theflexibility service, while the merchant is the entity providing the service.

- Affordability - The costs of implementing and using the system must be affordable for consumers and merchants, since these end-users are unlikely to be prepared to pay significantly extra to participate in Internet e-commerce transactions. For example, consumers are not willing to pay for a digital certificate in order to conduct e-commerce transactions although it is required in some e-payment scheme such a SET. Merchants will also not wish to invest significantly in engineering e-payment infrastructure.

- Reliability - The system must be reliable since it is used for the transmission and manipulation of sensitive information.

- Availability - The system must be available when needed.

- Speed of transaction - The transaction speed must be acceptable for e-commerce end-users.

- Interoperability - The system must be interoperable between different computing platforms, web browsers and server software packages in order to enable its use by the widest possible spectrum of e-commerce consumers and merchants.

E-C secure protocols are the widely recognizes logical operating standards for secure completion of information exchange, as well as the critical technique to ensure the confidentiality, integrity, authentication and non-repudiation of online transactions. Their completion serves as a key to provide online security. Internet E-C security protocol is the central research areas in $\mathrm{E}-\mathrm{C}$ as the endeavors to promote the development of E-C, and guarantee its security. The prevalent protocols are Security Socket Layer (SSL), Secure Electronic Transactions (SET) and 3D-Secure.

These protocols allow using cryptography to send confidential information on the Internet without being readable to malicious individuals. However, it turned out that these protocols are not as secure as we thought they would be. Indeed, several errors were discovered in cryptographic protocols after some years of use. The consequences that can generate vulnerability in a cryptographic protocol can be costly and irreversible for companies and individuals.
In this paper, we consider how E-commerce security requirements are fulfilled by our new protocol based on payment gateway and digital signature.

\section{RELATED WORK}

There have been many studies of E-commerce security. Security in E-commerce was described in the paper written by Dhilon [3] who introduce the stages to be provided for online purchase, the approach is based on encryption and compression for making information unreadable. However, Ecommerce security has become a consistent and growing problem as new internet technologies and application are developed; it needs new architecture to adapt to many changes. Al-SLamy [4] described the role of Pretty Good Privacy (PGP) to provide confidentiality, authentication, compression and segmentation services for E-commerce security. Byung Lee [5] introduced The Advanced Secure electronic payment (ASEP) which use ECC (Elliptic Curve Cryptosystem), SHA (Secure Hash Algorithm) and 3BC (Block Byte Bit Cipher) instead of RSA and DES in order to improve the strength of encryption and the speed of processing. Xuan Zhang [6] designed and implemented a new payment process to guarantee goods atomicity, certified delivery atomicity and protects sensitive information of cardholder and merchant.

Secure Sockets Layer (SSL) is a commonly used protocol used to encrypt messages between web browsers and web servers [7]. It encrypts the datagrams of the Transport Layer protocols. SSL is also widely used by merchants to protect the consumer's information during transmission, such as credit card numbers and other sensitive information. SSL is used to provide security and data integrity over the Internet and thus plays an important role. SSL has now become part of Transport Layer Security (TLS), which is an overall security protocol. One of the major problems of SSL is that the merchant can store the sensitive information of the cardholder, and the protocol does not prevent the non repudiation because the client authentication is optional.

SET (Secure Electronic Transaction) come to resolve the weakness of SSL in authentication and protection of sensitive information, SET ensures payment integrity, confidentiality and authentication of merchants and cardholders [8]. But SET is characterized by the complexity and the cost supported by the merchant (compared to the alternative proposed by SSL) because of the logistics of certificates distributing and client software installation, also it's difficult to manage nonrepudiation. To deal with it, VISA introduce 3D-secure [9], this protocol is based on the introduction of additional control when buying online in addition to the classic sensitive cardholder data. The customer validates the payment in new window by entering a secret data agreed with its own bank (password, date of birth, code received by SMS or generated by a personal drive).

\section{Secure Electronic Payment Protocol Design}

Our main idea is to design a secure and efficient protocol to protect online payment transactions against the fraud without involving the third party, our protocol respond to the requirements of e-payment security: confidentiality, integrity, authentication and non-repudiation. 


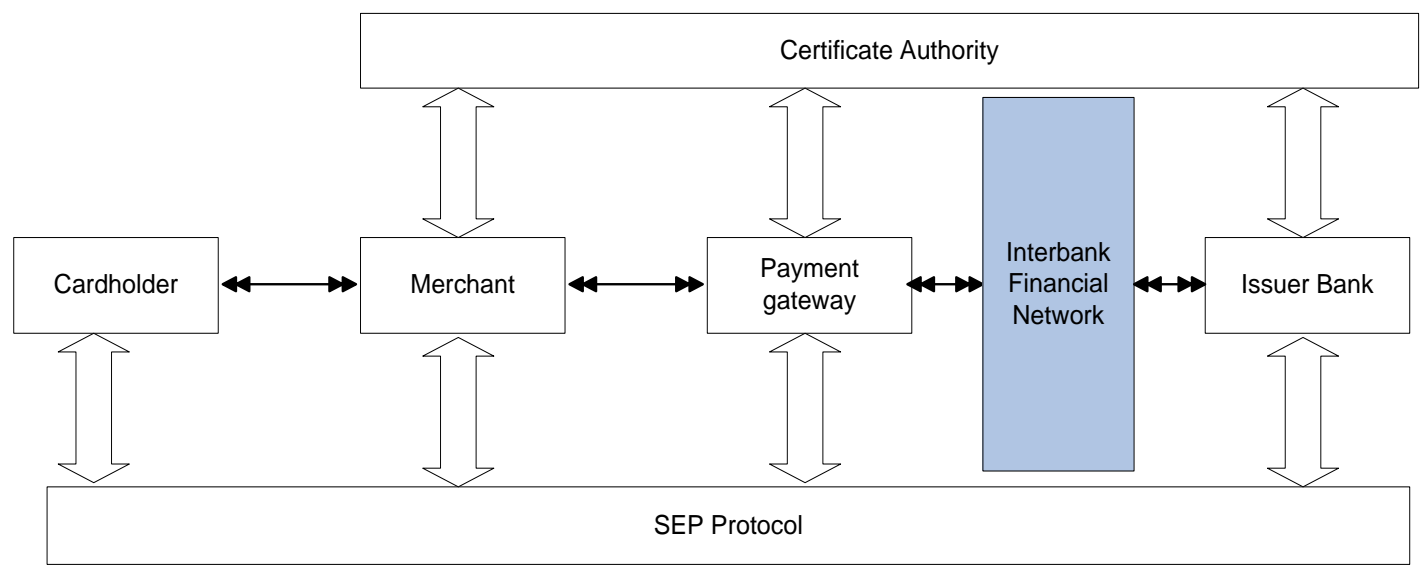

Fig. 1. Entities in SEP protocol

Our Secure Electronic Payment (SEP) protocol avoids the complexities relating to the implementation unlike SET and 3D-secure, integration and utilization are also easier than before.

For the convenience of written expression, we use the following notational conventions in this paper.

\section{$\mathrm{C}:$ Cardholder}

$\mathrm{M}$ : Merchant

PG : Payment Gateway

IB : Issuer Bank or Cardholder Bank

CA: Certificate authority

Vshop : Virtual Shopping Site

PAN: Card Number

CVV2: Card Verification Value or Crypto (three digits)

ExD: Expiry date of the card

OI: Order Information

PI: Payment Instructions

OIMD: OI Message Digest

PIMD: PI Message Digest

POMD: Payment Order Message Digest

K: Symmetric key generated randomly

Kum: Public key of merchant

Kupg: Public key of payment gateway

Kuis: Public key of issuer bank

Krm: Private key of merchant

Krpg: Private key of payment gateway

Kris: Private key of issuer bank

S: Sign

E: Encrypt

D: Decrypt

V: Verify signature

$\mathrm{H}$ : Hash

I: Concatenation

\#: Disconnect

Eq: Equal

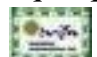

\section{: Certificate}

Our SEP protocol includes the following entities (see figure 1).

The standard description of SEP is illustrated in figure 2.

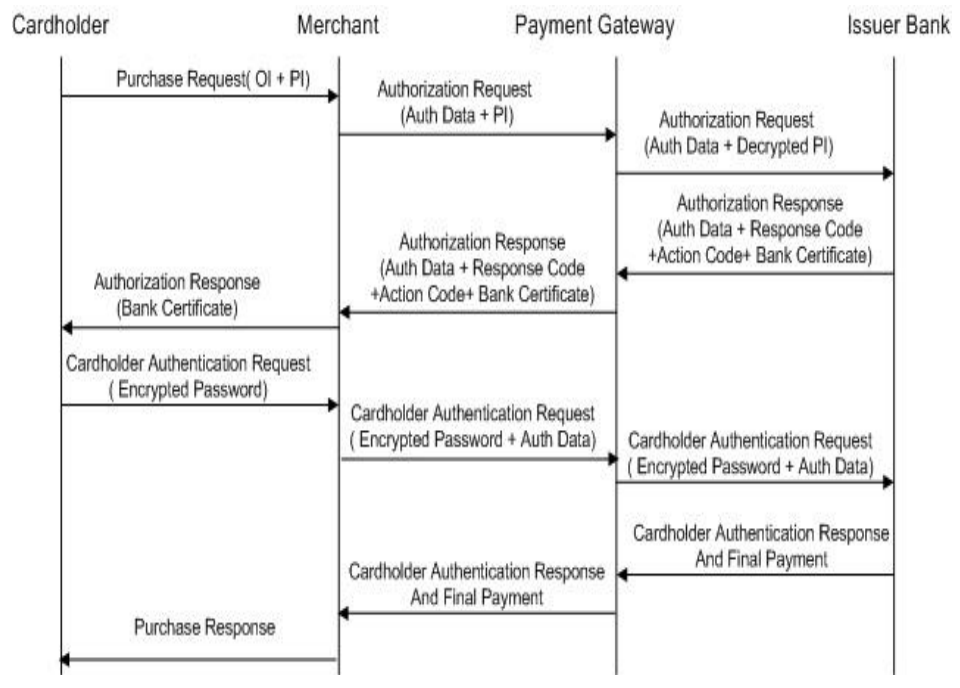

Fig. 2. Description of SEP protocol

\section{A. Registration process}

Merchant, payment gateway and issuer bank should register and obtain certificates from certificate authority (CA) before they involve in the SEP transaction.

Cardholder should register and obtain a password from his issuer bank (IB) before he involve in the SEP transaction. Purchase Request

Cardholder browses for items, select items to be purchased from the Vshop and get an order which contain the list of items to be purchased. Before stating purchase the cardholder and the merchant agree upon the order description amount. The cardholder then sends to the merchant his local ID and a fresh random challenge. The purpose of this is to give the cardholder with the merchant's signature certificate and the payment encryption certificate.

1) Cardholder generates OI, encrypted PI and dual signature. The dual signature is encrypted under a symmetric key generated randomly for the encryption; the cardholder is not requested to have his own certificate (see figure 3).

2) Cardholder prepares the purchase request and sends it to the merchant (see figure 4). 


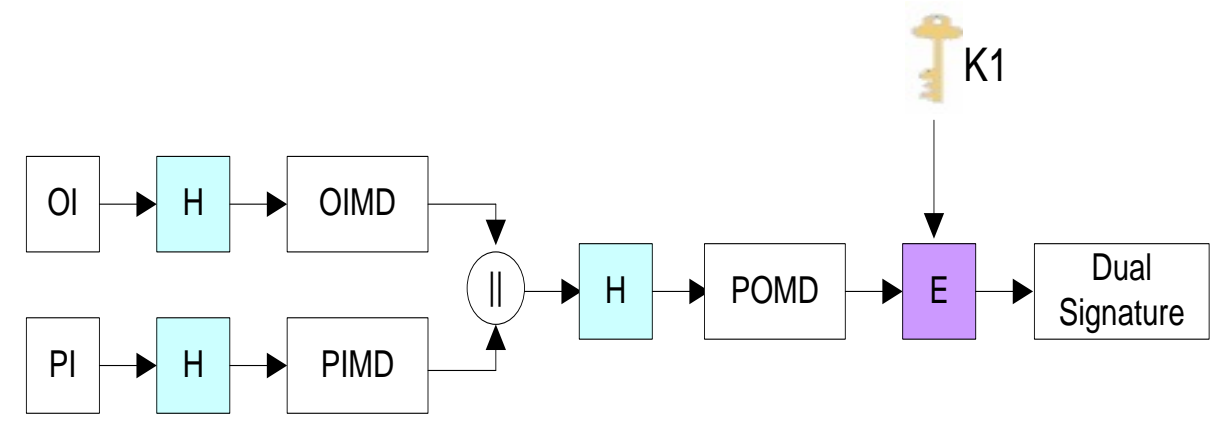

Fig. 3. Dual signature

3) The merchant extract the symmetric key, process the OI and transmit the encrypted PI to the payment gateway. (see figure 5)

\section{B. Authorization Request}

1) Merchant signs and sends authorization request to payment gateway, he sends the symmetric key Kl used for dual signature, the encrypted PI. The authorization request is encrypted under a symmetric key generated randomly. The payment gateway verifies the dual signature and gets PI. (see figure 5)

2) The payment gateway transmits the authorization with PI to the issuer bank through a secure and private interbank financial network. (See figure 6)

\section{Authorization Response}

1) The issuer bank verifies PI, verifies authorization request and run some issuer controls to check if the cardholder is allowed to make this transaction.

2) The issuer sends an authorization response and issuer bank certificate to the payment gateway through the secure interbank financial network (see figure 7). The authorization response contains the response code and the action code. The response code indicates if the authorization request is approved or no, the action code indicates if the cardholder is asked to be authenticated using his password. The purpose of this step is to give the cardholder with issuer bank encryption certificate.

3) The payment gateway signs and sends the authorization response and issuer certificate to the merchant. (see figure 8). The merchant check the action code, if the action code equals to ' $Y$ ' witch mean that the cardholder should be authenticated then, the merchant sends an authentication request to the cardholder containing the issuer certificate and some authorization data(see figure 9).

\section{Cardholder Authentication Request}

1) The cardholder verifies the issuer certificates and sends his personal password encrypted under the symmetric key. (see figure 10)

2) The merchant verifies the authorization data and transmit the encrypted password to the payment gateway. (See figure 11)

3) The payment gateway verifies authorization data, the hash of the encrypted password and transmits the encrypted password to the issuer for verification. The issuer decrypts the encrypted password and checks if is it the correct one for this cardholder. (see figure 12)

\section{E. Cardholder Authentication Response and final payment}

1) The issuer bank decrypts and verifies the password code, ensures the consistency between the authorization request and cardholder authentication request, debits the cardholder account and sends a payment response to the payment gateway. (see figure 13)

2) Finally the payment gateway transmits the payment response to the merchant (see figure 14). Merchant verifies the response and ships the good to the cardholder.

\section{F. SEP and Information Confidentiality}

For each step of transmission a symmetric key is generated randomly to encrypt electronic payment data. The encryption prevents the illegal information access and information stealing in transmission.

\section{G. SEP and Authentication}

$\checkmark$ Cardholder authenticates merchant and issuer bank

$\checkmark$ Merchant authenticates payment gateway and issuer bank

$\checkmark$ Payment gateway authenticates merchant and issuer bank

$\checkmark \quad$ Issuer bank authenticates cardholder using the password code.

\section{H. SEP and Information Integrity}

Data integrity is ensured by using MACs (Message Authentication Code) based on hash functions MD5 (16 bytes) or SHA-1 (20 bytes). The MAC is sent for every message transmitted between ecommerce actors.

\section{SEP and Non-Repudiation}

The non-repudiation property is guarantee by using the password code during the cardholder authentication request.

The issuer bank authenticates the card and the cardholder, so the cardholder cannot deny the fact that he had sent information afterwards. 

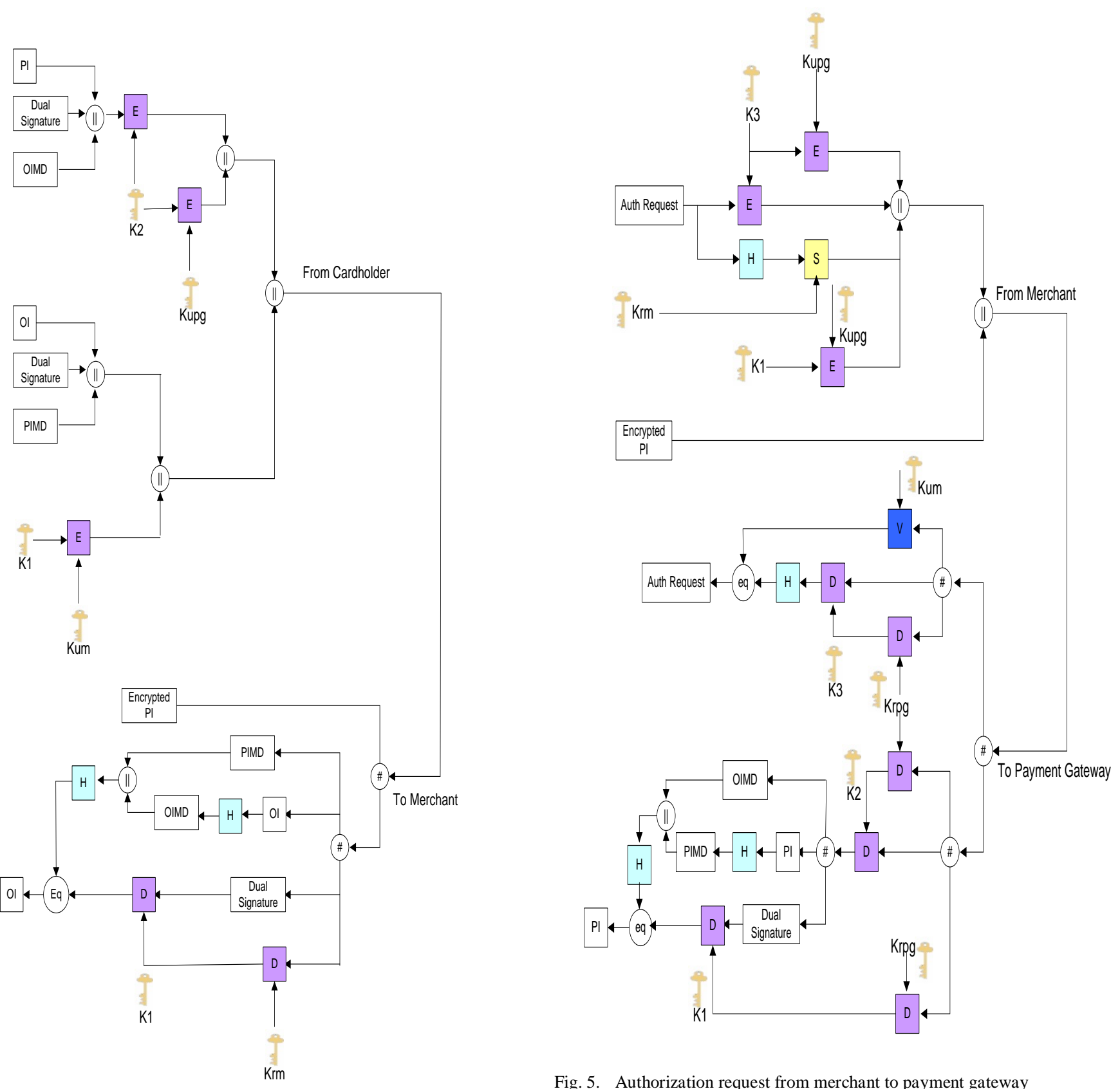

Fig. 5. Authorization request from merchant to payment gateway

Fig. 4. Purchase request from cardholder to merchant 


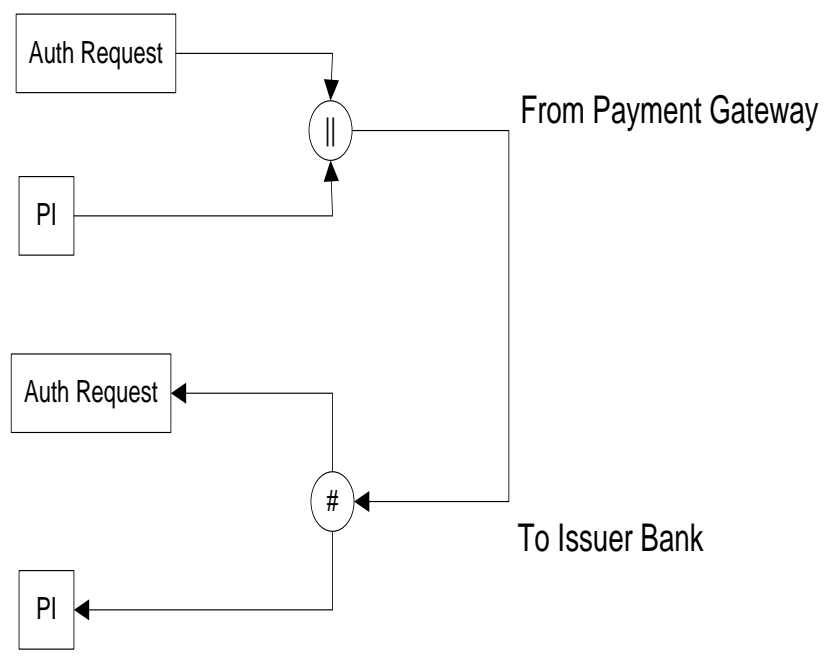

Fig. 6. Authorization request from payment gateway to issuer bank

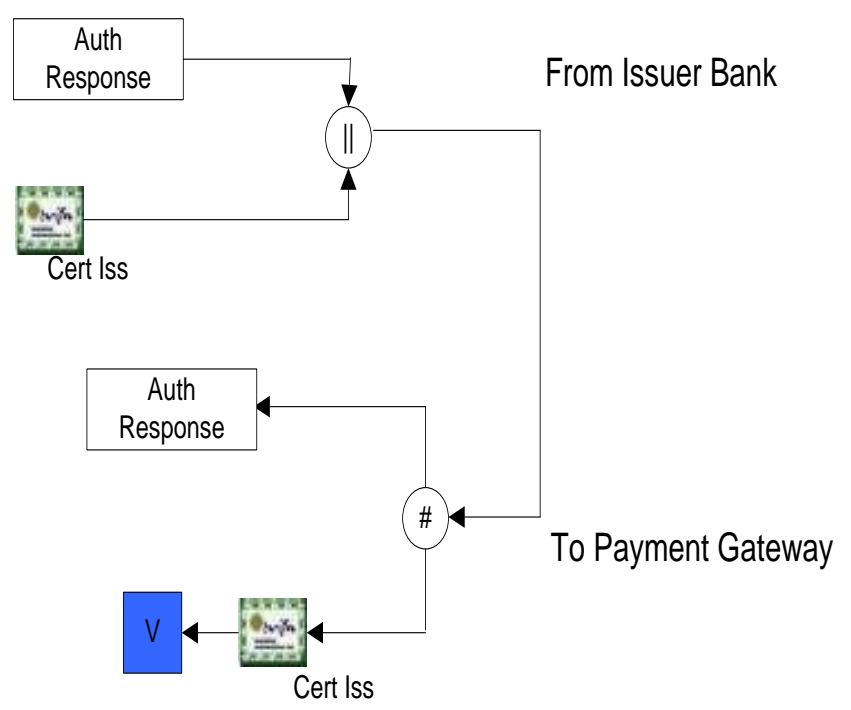

Fig. 7. Authorization response from issuer bank to payment gateway

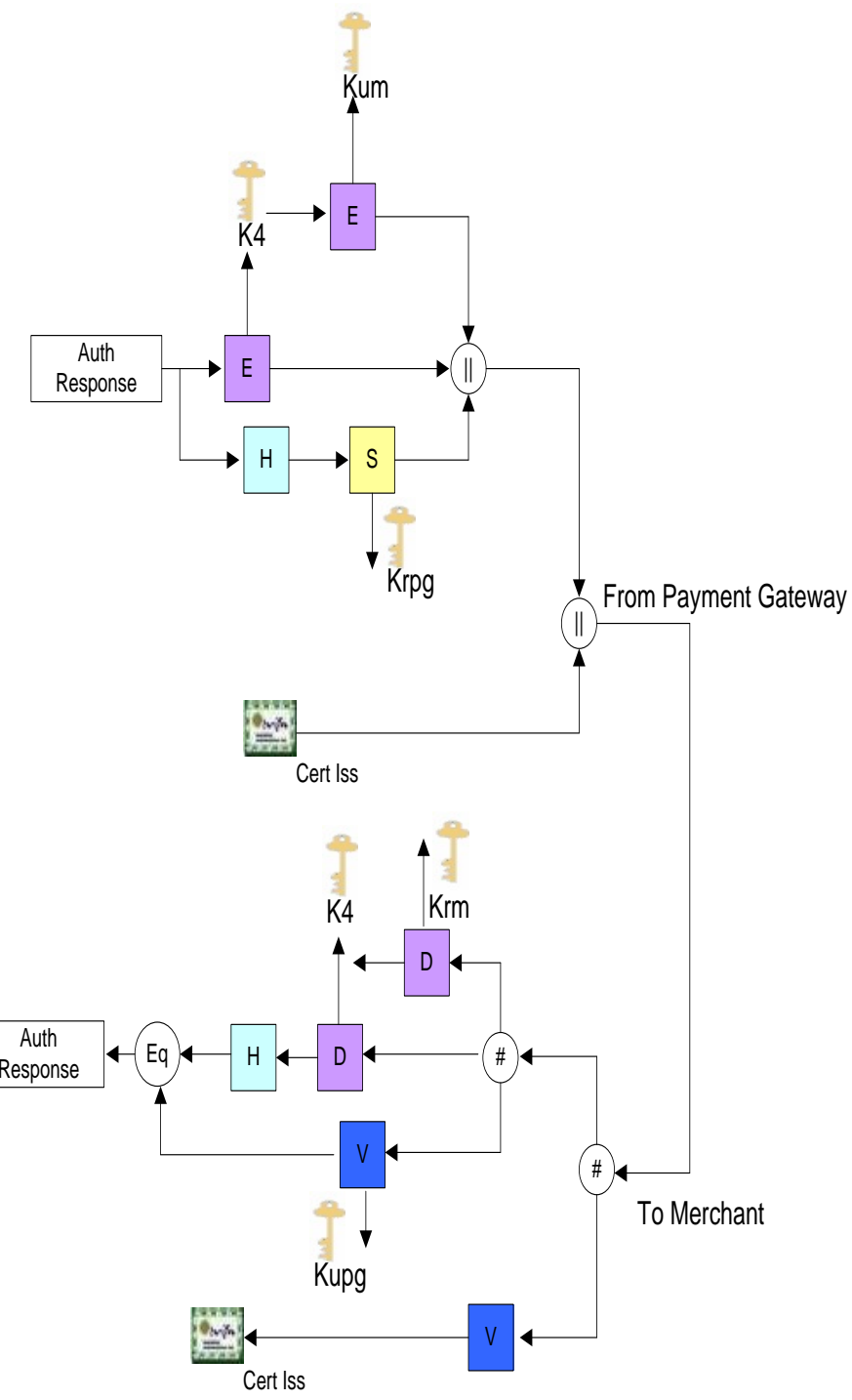

Fig. 8. Authorization response from payment gateway to merchant 


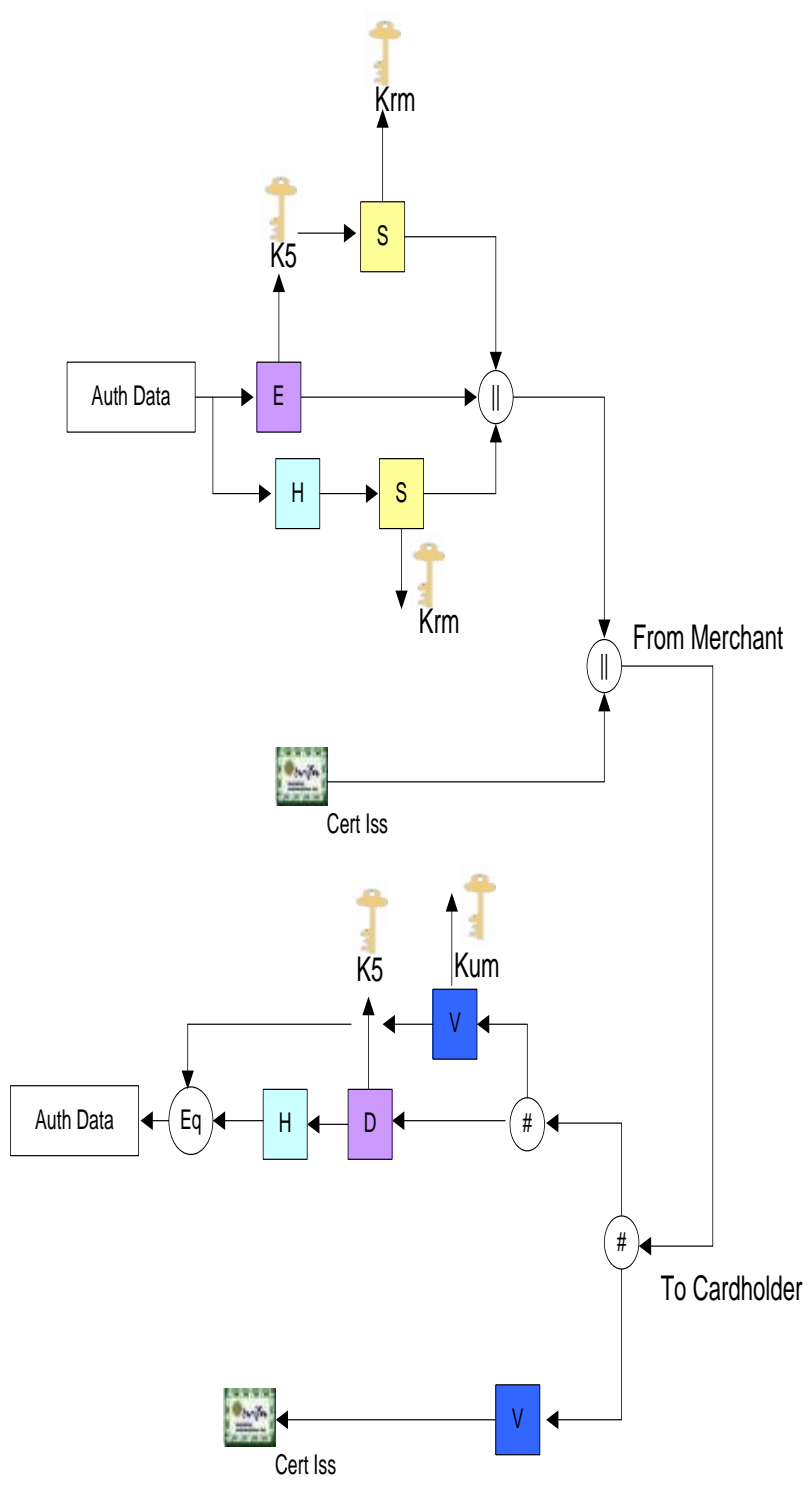

Fig. 9. Authorization response from merchant to cardholder

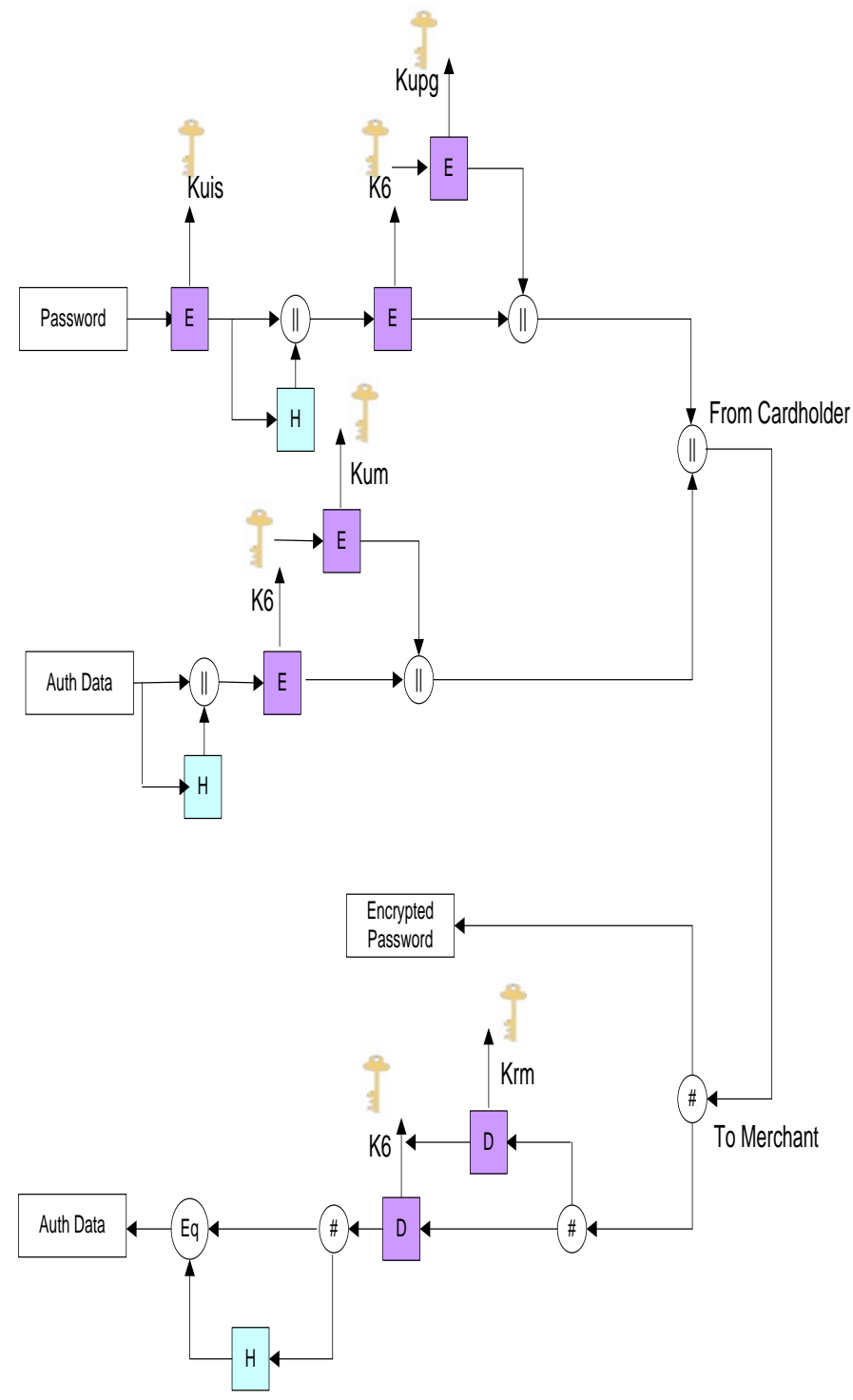

Fig. 10. Cardholder authentication request from cardholder to merchant 


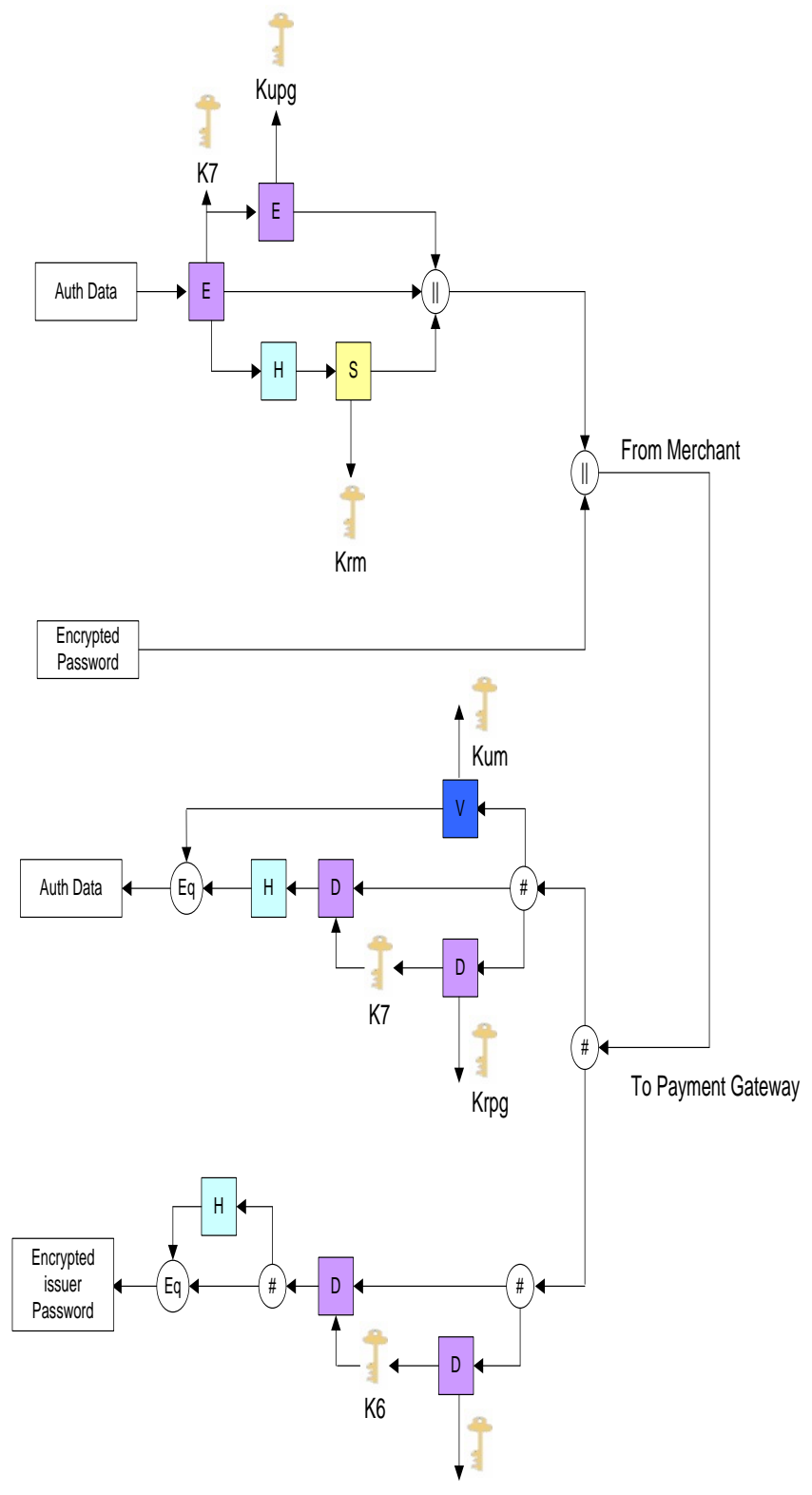

Fig. 11. Cardholder authentication request from merchant to payment gateway

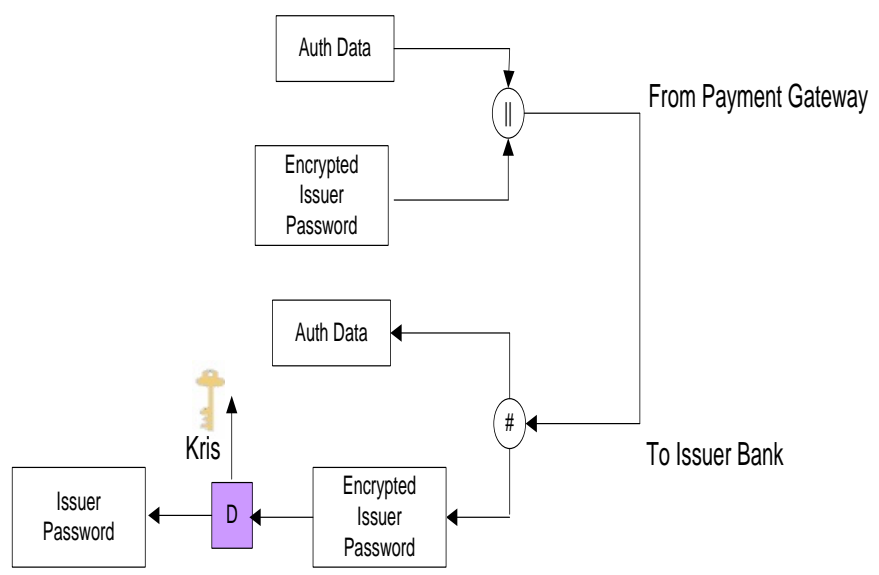

Fig. 12. Cardholder authentication request from payment gateway to issuer

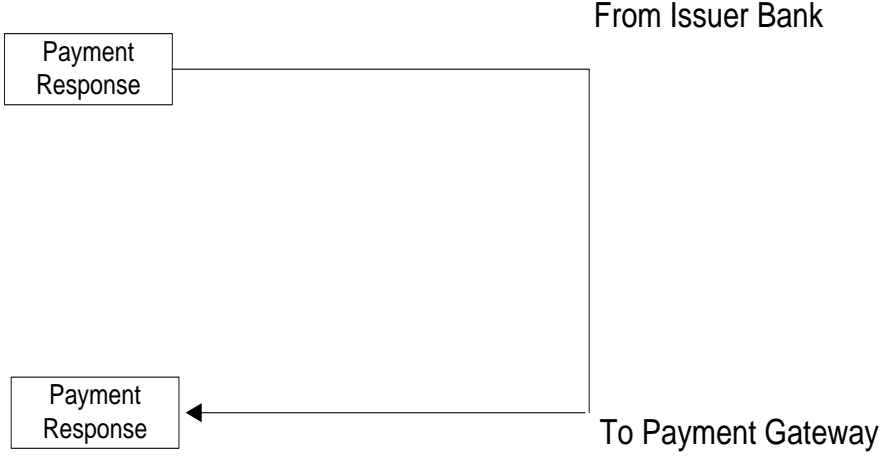

Fig. 13. Payment response from issuer to payment gateway
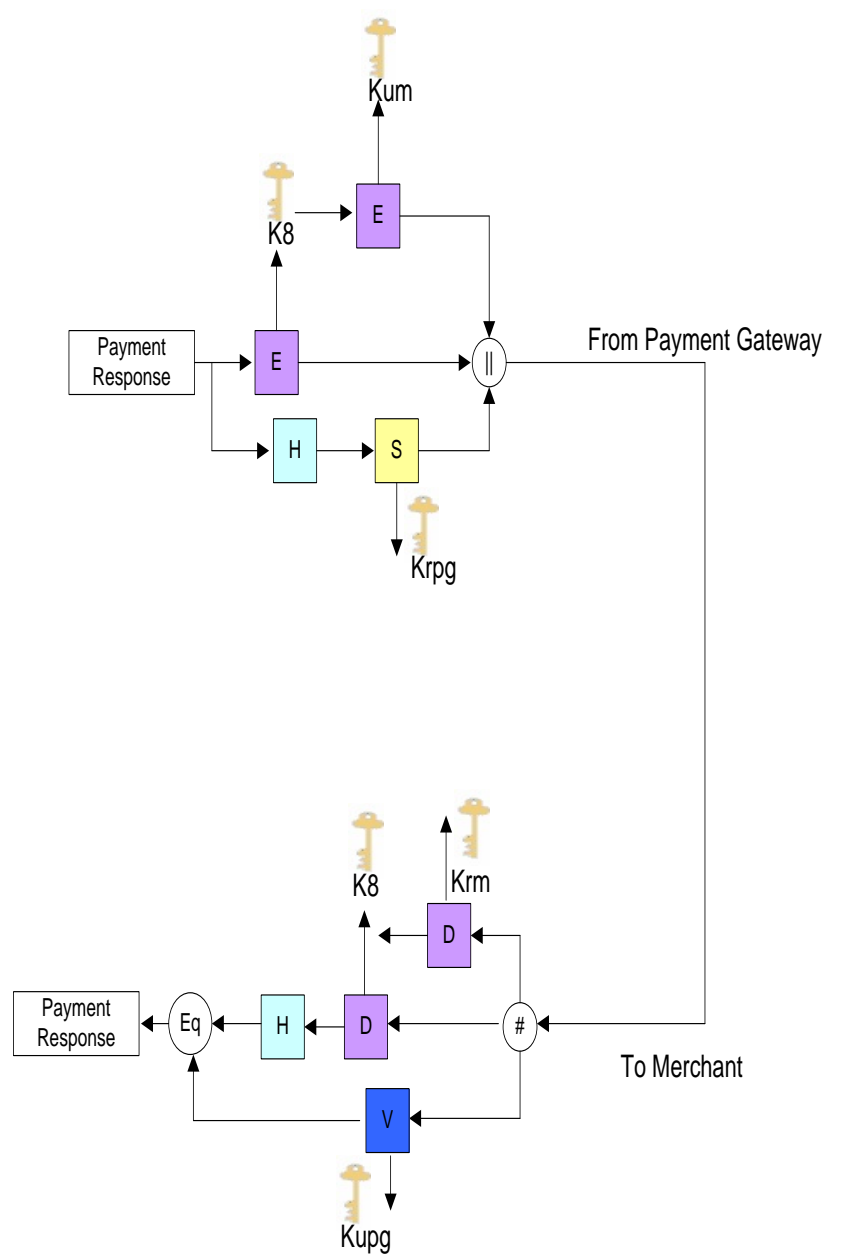

Fig. 14. Payment response from payment gateway to merchant

\section{J. SEP and End-user Implementation Requirements}

$\checkmark$ Usability: cardholder, merchant needs to install a special plug. The initialization process is so simple, since the cardholder does not need to have his certificate.

$\checkmark \quad$ Flexibility: SEP protocol have the desirable property that it can be used from any PC, as is currently the case for e-commerce transactions relying simply on 
SSL/TLS for cardholder- merchant communication security.

$\checkmark$ Affordability: if we compare SEP with 3D-Secure, 3D-Secure needs more investment in term of connectivity with VISA and ACS setup cost, also the merchant should be able to manage the cardholder authentication redirection to VISA. SEP needs just the attribution of security certificates to merchant, payment gateway and issuer bank, and plug-in setup.

$\checkmark$ Reliability: Of course, whilst the presence of incorrect functionality in security critical elements of SEP protocol is unlikely, there is still a significant possibility that accidental vulnerabilities will be present in implementation. Past experience indicates that it is very difficult to produce software which does not possess vulnerabilities exploitable by malicious software.

$\checkmark$ Availability: Unlike 3D-Secure, for SEP protocol card issuers and acquirers are not required to implement any system with VISA. Once the issuer has the software, they can support SEP transactions. Equally, consumer will be happy to perform a simple registration process to get the password coder and install the plug-in, no security certificate is needed.

$\checkmark$ Speed of transaction: SEP protocol employs DES for symmetric encryption and RSA for certificate verification. The issuer verification of cardholder identity is an important factor for transaction performance. The SEP protocol avoid the complexity of 3D-Secure related to Visa directory. It's difficult to decide about transaction speed because it's related also to networking speed and server's performance. $\checkmark$ Interoperability: SEP plug-ins can be installed on the consumer PC easily, so interoperability issues are less likely to arise.

\section{CONCLUSION}

SEP protocol is a good transaction protocol for credit card payment. In this paper we improved how well SEP protocol meets the e-payment security requirements and identified enduser implementation requirement. A future research topic is to analysis the security and the performance of our protocol.

\section{REFERENCES}

[1] HASSLER, V. (2001). SECURITY Fundamentals FOR E-COMMERCE. ARTECH HOUSE, MASSACHUSETTS

[2] Z. Jiemiao, Research on E-Payment Protocol, Information Management, Innovation Management and Industrial Engineering (ICIII), 2011, pages $121-123$

[3] G. Dhillon, J. Ohri, Optimizing Security in E-commerce through Implementation of Hybrid Technologies, CSECS'06 Proceedings of the 5th WSEAS International Conference on Circuits, Systems, Electronics, Control \& Signal Processing, Pages 165 - 170.

[4] A.A. Slamy, E-Commerce security, IJCSNS International Journal of Computer Science and Network Security, VOL.8 No.5, May 2008

[5] B. Lee, T.Lee, An ASEP (Advanced Secure Electronic Payment) Protocol Design Using 3BC and ECC(F2m) Algorithm, e-Technology, e-Commerce and e-Service, 2004. EEE '04. 2004 IEEE International Conference on, pages $341-346$

[6] X. Zhang, Implementation of a Suggested E-commerce Model Based on SET Protocol, Software Engineering Research, Management and Applications (SERA), 2010 Eighth ACIS International Conference on, pages $67-73$

[7] A.Craft, T A and R. Kakar, E-Commere Security, Conference on information systems Applied Research 2009, v2 Washington.

[8] H. Houmani, M. Mejri, Formal Analysis of SET and NSL Protocols Using the Interpretation Functions-based Method, Journal of Computer Networks and Communications Volume 2012, Article ID 254942, page 18

[9] P. Jarupunphol, C. Mitchell, Measuring 3-D Secure and 3D SET against e-commerce end-user requirements, Proceedings of the 8th Collaborative electronic commerce technology and research conference (CollECTeR (Europe) 2003), National U 